\title{
Endoscopic ultrasound-guided hepaticogastros- tomy using two metal stents locked together
}

Endoscopic ultrasound-guided hepaticogastrostomy (EUS-HGS) is a recently developed technique for biliary decompression in patients with biliary obstruction, which offers an alternative when endoscopic biliary drainage (EBD) or percutaneous transhepatic biliary drainage (PTBD) fail, or when the papilla is inaccessible [1]. However, EUS-HGS has several disadvantages, including early stent occlusion by granulation tissue, which can develop when there is a mismatch in the bore diameter between the bile duct and the stent, and stent migration, which may be fatal [2] and could be due to the use of a metal stent of insufficient diameter.

Recently, a novel partially covered metal stent (bare-end type, Niti-S covered biliary stent; TaeWoong Medical, Seoul, Korea; Century Medical, Tokyo, Japan) has been made available ( $\bullet$ Fig. 1 ). This stent is $12 \mathrm{~cm}$ long, having $1 \mathrm{~cm}$ that is uncovered at the top, with the opposite end being flared. However, it may still be difficult to avoid the problems of early stent occlusion and possible stent migration. Therefore, we describe a novel technique for EUS-HGS, the "locking stent" method, which may overcome these difficulties.

A 78-year-old woman was admitted to our hospital with obstructive jaundice due to pancreatic cancer and liver metastases. On laboratory examination, biochemistry tests yielded the following abnormal results: total bilirubin $18.2 \mathrm{mg} / \mathrm{dL}$, alanine aminotransferase $218 \mathrm{U} / \mathrm{L}$, aspartate aminotransferase $238 \mathrm{U} / \mathrm{L}$, and alkaline phosphatase 1882 U/L. An attempt was made at EBD; however, because of tu-

\section{Video 1}

Endoscopic ultrasound-guided hepaticogastrostomy (EUS-HGS) being performed showing stenosis in the middle of the common bile duct. An uncovered metal stent is first deployed between the left intrahepatic bile duct and the periphery of the B3 segment. A second partially covered metal stent is then positioned with its bare end within the end of the first stent in the B3 segment (so that the two stents are locked together) and its other end in the stomach. mor invasion into the duodenum, we were unable to access the papilla.

We therefore decided to perform EUSHGS. The intrahepatic bile duct (segment 3: B3) was punctured using a 19G needle (Sono Tip Pro Control 19G; Medi-Globe GmbH, Rosenheim, Germany). A 0.025inch guidewire (VisiGlide, angle type; Olympus Medical Systems, Tokyo, Japan) was then passed through the intrahepatic bile duct ( $\bullet$ Fig. 2 a). We initially inserted an uncovered stent $(8 \mathrm{~mm} \times 8 \mathrm{~cm}$, Niti-S LCD; TaeWoong Medical) between the left intrahepatic bile duct and the periphery of the B3 segment ( Fig.2b). We then deployed a second stent (the bareend, $10-\mathrm{mm} \times 12-\mathrm{cm}$, covered biliary stent) with its end part way inside the first uncovered stent ( Fig.2c, Video 1). Therefore, we had two linked stents that were "locked together" running between the upper common bile duct and the stomach ( Fig. 2d). The patient died 5 months later, but during this time no stent dysfunction was seen.

We believe that this "locking stent" method offers a safe way to prevent stent migration and obstruction. Because the bare end of the second stent locks into the first uncovered stent, the hepaticogastrostomy stent is unlikely to migrate into the intrahepatic bile duct or the stomach. Additionally, because the distal hepaticogastrostomy stent is placed in the left bile duct, mismatch of the bore diameters between the bile duct and the stent is unlikely to cause the formation of granulation tissue. Although validation is required in a prospective clinical trial, our technique appears to be capable of safely and effectively preventing stent dysfunction.

Endoscopy_UCTN_Code_TTT_1AS

Competing interests: None

\section{Takeshi Ogura, Daisuke Masuda, Akira Imoto, Rieko Kamiyama, Kazuhide Higuchi}

Second Department of Internal Medicine, Osaka Medical College, Osaka, Japan

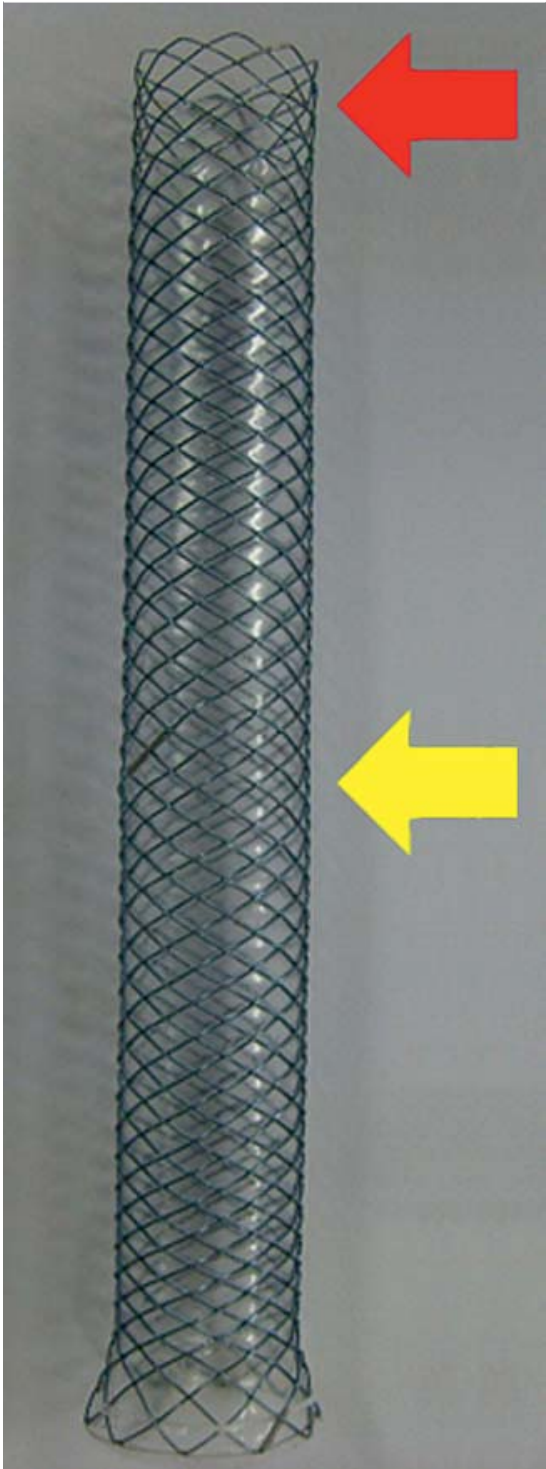

Fig. 1 The bare-end type, Niti-S biliary covered metal stent, which has the top $1 \mathrm{~cm}$ of the stent uncovered (red arrow), while the remainder of the stent is fully covered. 

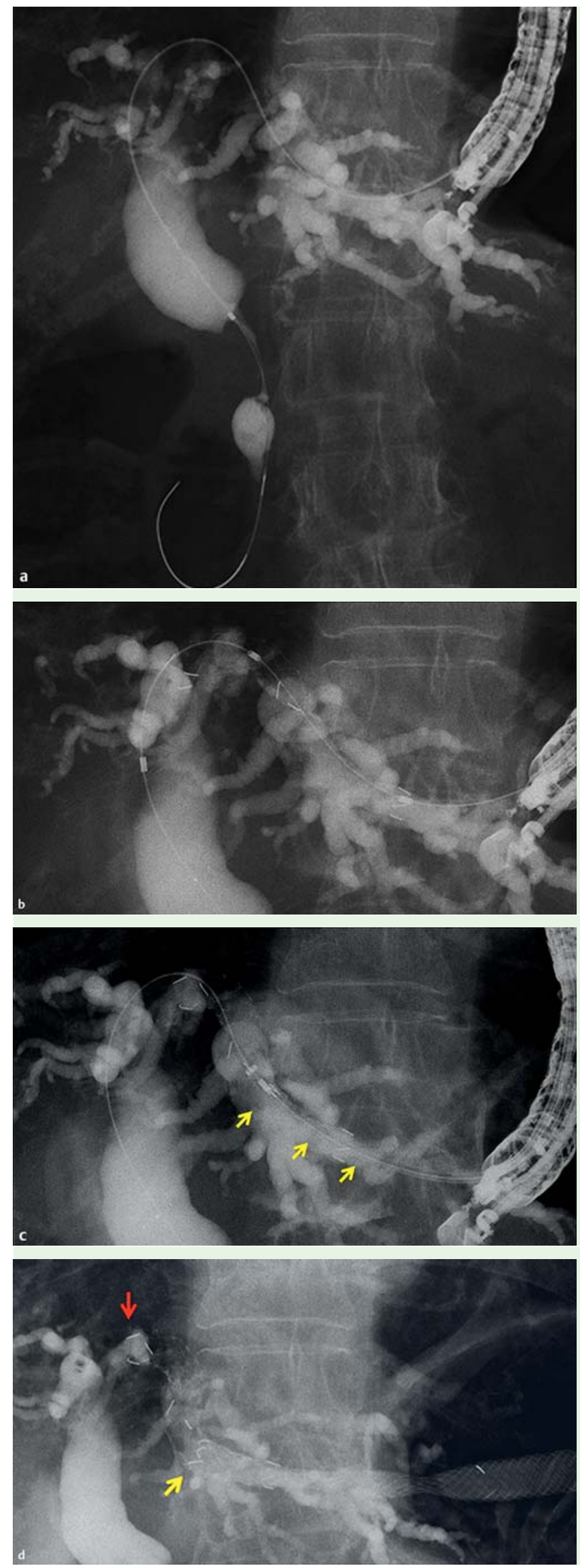

Fig. 2 Endoscopic ultrasound-guided hepaticogastrostomy (EUS-HGS) in a 78-yearold woman with pancreatic cancer. a Obstruction to the middle of the common bile duct and a guidewire that has been inserted from the stomach through into the intestine via the intrahepatic bile duct. b An uncovered metal stent in position between the left intrahepatic bile duct and the periphery of the $B 3$ segment. c The delivery system of a second stent (the new bare-end covered biliary stent) positioned for insertion with the bare end of this stent inside the first uncovered metal stent. d The two metal stents running between the upper common bile duct and the stomach with the partially covered metal stent (yellow arrow) locked together with the uncovered metal stent (red arrow).

\section{References}

1 Giovannini M, Dotti M, Boris E et al. Hepaticogastrostomy by echoendoscopy as a palliative treatment in a patient with metastatic biliary obstruction. Endoscopy 2003; 35: 1076-1078

2 Martin FP, Rossini LG, Ferrari AP. Migration of a covered metallic stent following endoscopic ultrasound-guided hepaticogastrostomy: fatal complication. Endoscopy 2010; 42: E126-E127

\section{Bibliography}

DOI http://dx.doi.org/

10.1055/s-0033-1359191

Endoscopy 2014; 46: E65-E66

(c) Georg Thieme Verlag KG

Stuttgart · New York

ISSN 0013-726X

\section{Corresponding author \\ Takeshi Ogura, MD, PhD}

Second Department of Internal Medicine Osaka Medical College

2-7 Daigaku-machi

Takatsuki-shi

Osaka 569-8686

Japan

Fax: +81-72-6846532

oguratakeshi0411@yahoo.co.jp 